\section{Rectal and Bladder Dose Measurements in the Intracavitary Applications of Cervical Cancer Treatment with HDR Afterloading System: Comparison of TPS Data with MOSFET Detector}

\author{
Singh N. ${ }^{1 \oplus}$, Ahamed S. ${ }^{2,3}$, Sinha A. ${ }^{4 * \odot}{ }^{\oplus}$, Srivastava S. ${ }^{5}$, \\ Painuly N. K. ${ }^{1}$, Mandal A. ${ }^{6}$, Prasad S. N. ${ }^{7}$
}

\begin{abstract}
Background: Intracavitary brachytherapy plays a major role in management of cervical carcinoma. Assessment of dose received by OAR's therefore becomes crucial for the estimation of radiation toxicities in HDR brachytherapy.

Objective: Purpose of this study is to evaluate the role of in vivo dosimetry in HDR brachytherapy and to compare actual doses delivered to OAR's with those calculated during treatment planning.
\end{abstract}

Material and Methods: In this retrospective study, 50 patients of cervical carcinoma were treated by Microselectron HDR. Out of 50 patients, 26 were treated with a dose of $7 \mathrm{~Gy}$ and 24 with a dose of $9 \mathrm{~Gy}$, prescribed to point A. Brachytherapy planning and evaluation of dose to bladder and rectum was done on TPS \& in vivo dosimetry was performed using portable MOSFET.

Results: Calibration factors calculated for both dosimeters are almost equal and are $0.984 \mathrm{cGy} / \mathrm{mV}$ and $1.0895 \mathrm{cGy} / \mathrm{mV}$. For bladder, dose deviation was found to be within $\pm 5 \%$ in 28 patients, $\pm 5-10 \%$ in 14 patients, $\pm 10-15 \%$ in 4 patients. Deviation between TPS-calculated dose and dose measured by MOSFET for rectum was within $\pm 5 \%$ in 31 patients, $\pm 5-10 \%$ in 8 patients, and $\pm 10-15 \%$ in 7 patients.

Conclusion: TPS calculated doses were slightly higher than that measured by MOSFET. The use of small size MOSFET dosimeter is an efficient method for accurately measuring doses in high-dose gradient fields typically seen in brachytherapy. Therefore, to reduce the risk of large errors in dose delivery, in vivo dosimetry can be done in addition to TPS computations.

Citation: Singh N, Ahamed S, Sinha A, Srivastava S, Painuly NK, Mandal A, Prasad SN. Rectal and Bladder Dose Measurements in the Intracavitary Applications of Cervical Cancer Treatment with HDR Afterloading System: Comparison of TPS Data with MOSFET Detector. $J$ Biomed Phys Eng. 2020;10(2):141-146. doi: 10.31661/jbpe.v0i0.1065.

\section{Keywords}

MOSFET; ICRU; TPS; Brachytherapy; In Vivo Dosimetry; Radiation Dosimeters

\section{Introduction}

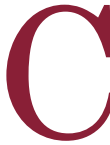

ancer of the cervix uteri forms one third of diseases leading to death among woman worldwide. Intracavitary brachytherapy (ICBT) in combination with external beam radiotherapy (EBRT) forms an essential part of radiotherapy. However the main concern is how to interpret parameters correlated with organs at risk (OAR) that
${ }^{1} \mathrm{PhD}$, Department of Radiotherapy, King George Medical University,

Lucknow 226003, Uttar

Pradesh, India

${ }^{2} \mathrm{MSc}$, Department of

Radiation Physics, MNJ

Institute of Oncology and

Regional Cancer Centre,

Hyderabad, 500004

Telangana

${ }^{3} \mathrm{MSc}$, Department of

Physics, Jawaharlal Nehru

Technological University,

Ananthapuramu, 515002 .

Andhra Pradesh

${ }^{4} \mathrm{PhD}$, Department of

Radiotherapy, J.K.Cancer

Institute, Kanpur 208002

Uttar Pradesh, India

${ }^{5} \mathrm{MSc}$, Department of Ra-

diotherapy, King George

Medical University,

Lucknow 226003, Uttar

Pradesh, India

${ }^{6} \mathrm{PhD}$, Department of

Radiotherapy and Radia-

tion Medicine, Institute

of Medical Sciences.

Banaras Hindu University,

Varanasi, 221005, India

${ }^{7} \mathrm{MD}$, Department of

Radiotherapy, J.K.Cancer

Institute, Kanpur 208002

Uttar Pradesh, India

*Corresponding author:

A. Sinha

Assistant Professor

(Radiological Physics)

Department of Ra-

diotherapy J.K.Cancer

Institute, Kanpur (UP)

208002 India

E-mail: ashutosh22jan@ gmail.com

Received: 5 December 2018 Accepted: 28 December 2018 
are pertinent to predict adverse results of brachytherapy $[1,2]$. In brachytherapy, International Commission on Radiation Units and Measurements (ICRU) proposed dose reference points for bladder and rectum to predict clinically relevant endpoints and bring uniformity in dose reporting [3].

Evaluation of dose recorded by OAR in high-dose rate brachytherapy becomes necessary for the estimation of radiation toxicity. Additionally, the measurement of delivered dose during first treatment session may be utilized to plan subsequent dose fractions with corrective measures. Any source of error in delivery of dose can be detected by the quality assurance procedure in place.

Recently with the newer imaging technology, the protocols for prescribing and reporting doses have changed. Drastic changes are due to 3-D dose-volume estimations and reporting [4-6] in comparison with the point based on dose calculation formalism proposed by ICRU 38 [3, 7]. However, apart from reporting, a number of parameters show effects of estimated dose even in the era of 3-D planning.

In ICBT, in vivo dosimetry plays a vital role to predict doses for bladder and rectum and also helps to avoid misadministration of planned dose. Thermoluminescence dosimeters (TLDs) and semiconductor diodes were chosen for in vivo dosimetry by many groups [8-12]. These dosimeters are mainly used to measure dose for specific points that may not always represent the clinically relevant dose received by OAR and hence they require repetition of dose measurement at various points. MOSFET dosimeters are commonly used for in vivo measurement of dose in real time and also estimate point doses more accurate than other dosimeters due to the small sensitive volume.

The goal of the present study was to assess the role of in vivo dosimetry with mobile MOSFET detectors in HDR Brachytherapy and compare the dose received by organs at risk (using in vivo procedures) with calculated dose using treatment planning system.

\section{Material and Methods}

In this retrospective study, 50 patients of cervical carcinoma were treated with Microselectron HDR. Out of 50 patients, 26 and 24 were treated with the dose of 7 and 9 Gy, respectively, prescribed to point A. Dose measurements with MOSFET were carried out for the first fraction of each patient using standard source loading pattern. All patients received high-dose rate ICRT along with EBRT during August 2017 and July 2018.

A combination of ovoid (half and full) and tandem angles $\left(15^{\circ}, 30^{\circ}\right.$ and $\left.45^{\circ}\right)$ of FletcherSuit model applicators (Nucletron) was used to suit patient's anatomy. Individualized gauze packing was done to immobilize the geometry of the applicators and distance bladder and rectum. Foley's catheter inserted into bladder was filled with $7 \mathrm{cc}$ of diluted contrast and pulled down to be seated at bladder trigone. A rectal probe containing five radio opaque markers was inserted in the rectum to visualize on the radiograph set. Anterior-posterior (AP) and lateral radiographs were acquired on the X-ray machine for brachytherapy planning. Catheter reconstruction was done on the radiographs and also bladder and rectal points were identified with ICRU 38. Point A was defined on AP radiograph at $2 \mathrm{~cm}$ superior to the flange and $2 \mathrm{~cm}$ lateral from the axis of the intrauterine tandem. Patients were prescribed either the dose of 18 Gy delivered over 2 fractions or 21 Gy delivered over 3 fractions at point A. Dwell times were optimized to minimize dose to bladder and rectal points.

Further for in vivo dosimetry, portable MOSFET (metal oxide semiconductor field effect transistor) dosimetry system with two MOSFETs (TN - 502RD-H, SN: 33545 \& 33546) of standard sensitivity and an electrometer TN RD 70W dose verification system (Team Best, Canada) were used for estimation of bladder and rectum dose. The MOSFET dosimeter is an electronic device that measures integrated 
radiation dose. The small sensitive volume of $1 \mathrm{~mm}^{2}$ with an active area of $0.2 \times 0.2 \mathrm{~mm}$ located under a black epoxy bulb is used for dosimetry in steep dose gradients.

The Oncentra TPS version - 4.5.2 was used for brachytherapy planning and evaluation of dose to the bladder and rectum. The dose calculation algorithm used in this study is based on the formalism provided by Task Group-43 (TG 43, American Association of Physicists in Medicine). Treatment plans were optimized to keep bladder and rectal doses, below $80 \%$ of the dose prescribed to point $\mathrm{A}$ or to the extent possible. However, the dose to either bladder or rectum was greater than $80 \%$ of dose at point $\mathrm{A}$ in few cases.

A Siemens Somatom Emotion 16 slice CT scanner was used to acquire CT images of cylindrical phantom used for the calibration of MOSFET detectors. For calibration of MOSFET detectors, an acrylic cylindrical phantom with a hole at center (for source) and four equidistant holes from center located peripherally at $0.75 \mathrm{~cm}$ at $0^{\circ}, 90^{\circ}, 180^{\circ}$ and $270^{\circ}$ angles with MOSFET dosimeters in place was scanned by $5 \mathrm{~mm}$ slice thickness and the CT data was exported to TPS. Plans were generated by a single dwell position of Ir-192 source at the centre of cylindrical phantom with prescribed doses of $50 \mathrm{cGy}, 100 \mathrm{cGy}, 200 \mathrm{cGy}$, $300 c G y, 500 c G y, 700 c G y$ and 900 cGy at 0.75 $\mathrm{cm}$ from the center. The dose-response curve was found to be linear for irradiation doses uptil 9 Gy.

\section{Results}

The dose response curve for one MOSFET (TN - 502RD-H, SN: 33545) until the dose of 900cGy is shown in Figure 1. Two MOSFETs used in this study were calibrated and the dose response relationship is linear. The calibration factors calculated for both the dosimeters are almost $0.984 \mathrm{cGy} / \mathrm{mV}$ and $1.0895 \mathrm{cGy} / \mathrm{mV}$, respectively.

The maximum absorbed dose measured using MOSFET detector \& calculated by TPS

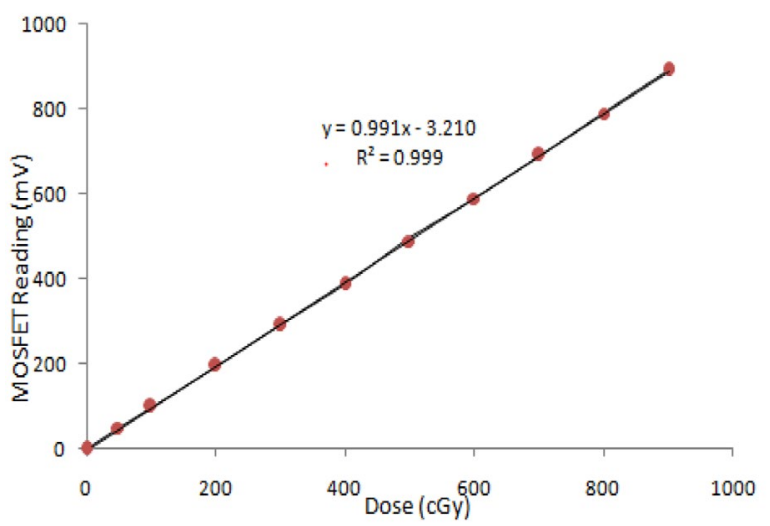

Figure 1: Calibration curve drawn between the prescribed dose and mobile MOSFET reading.

for bladder and rectum is shown in Figures 2 and 3 respectively. The mean dose estimated for the 50 patients, by TPS at the dose reference point placed in bladder is $6.13 \mathrm{~Gy} \pm 1$ and the dose measured by MOSFET placed in bladder is recorded as $6.01 \mathrm{~Gy} \pm 1$. For rectum, mean dose received by the TPS is 3.52 Gy \pm 0.7 whereas dose measured by the MOSFET placed in rectum is $3.40 \mathrm{~Gy} \pm 0.7$. For bladder, dose deviation was found to be within $\pm 5 \%$ in 28 patients, $\pm 5-10 \%$ in 14 patients, $\pm 10-15 \%$ in 4 patients. For four patients the dose deviation was over $\pm 15 \%$ [Figure 4]. The deviation between the TPS-calculated dose and the dose measured by MOSFET for rectum was within

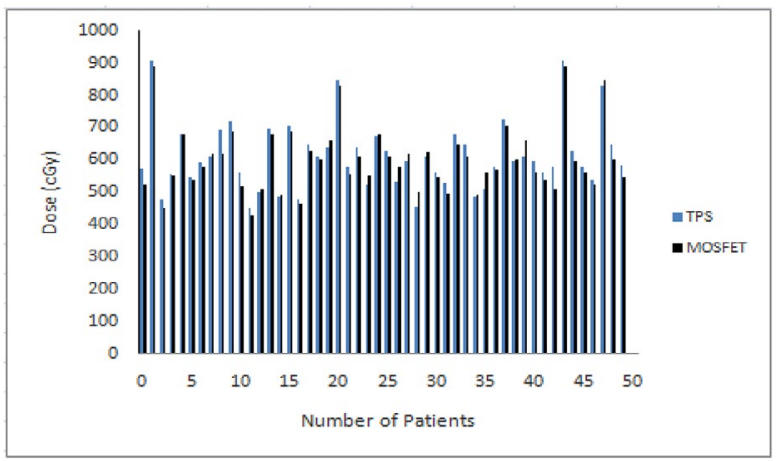

Figure 2: Comparison of bladder dose measured using a MOSFET Detector and calculated by TPS 
$\pm 5 \%$ in 31 patients, $\pm 5-10 \%$ in 8 patients, and $\pm 10-15 \%$ in 7 patients. Four patients recorded dose deviation exceeding $\pm 15 \%$ [Figure 5].

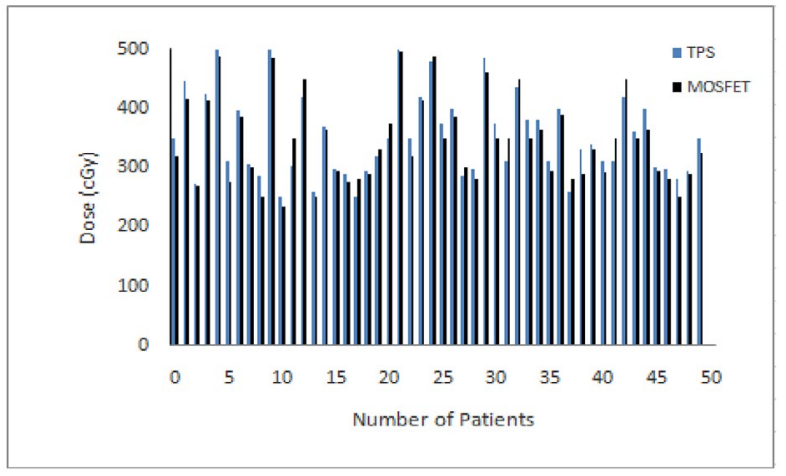

Figure 3: Comparison of rectal dose measured using a MOSFET Detector and calculated by TPS.

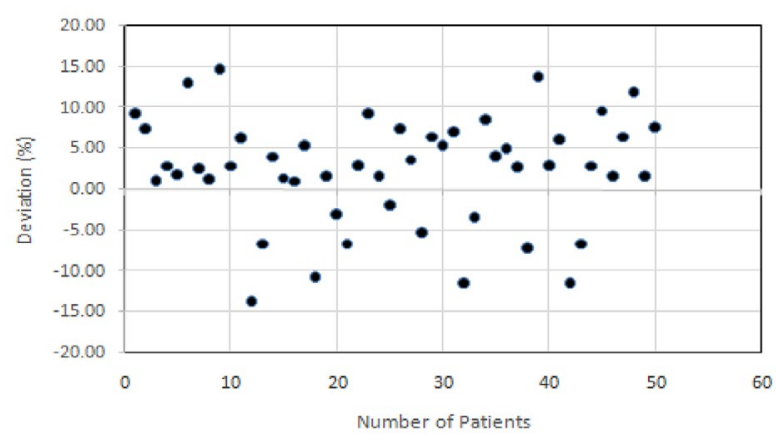

Figure 4: Figure shows the percentage deviation between the TPS dose and MOSFET dose for Bladder.

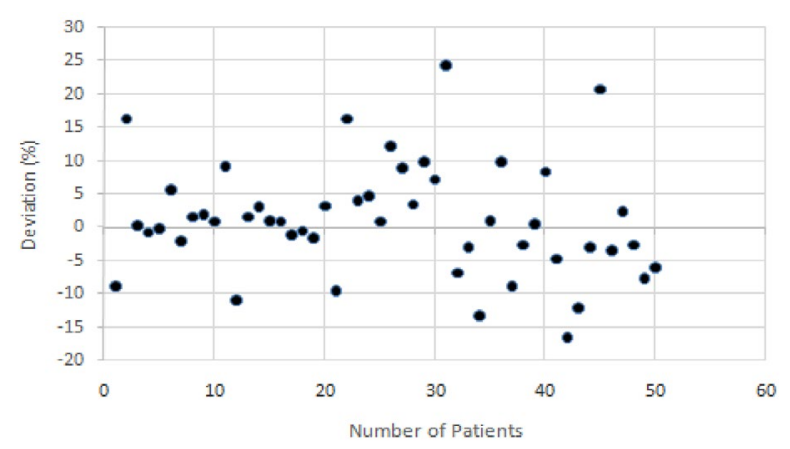

Figure 5: Figure shows the percentage deviation between the TPS dose and MOSFET dose for Rectum.
The data obtained from TPS and MOSFET was analyzed by the means of One - Way ANOVA using SPSS statistical software, version 20. No statistical significance was found between dose estimated by TPS and MOSFET for bladder and rectum, as p-value $>0.05$.

\section{Discussion}

In the present study, we used MOSFET dosimeters for measurement of rectum and bladder dose according to ICRU 38. MOSFET dosimeters in external beam therapy are well documented; however, there are limited studies in brachytherapy applications. In intracavitary application, the point of dose measurement is placed in the high-dose gradient regions and therefore placement of the detector in the correct position is extremely important. In vivo dose measurements were compared with calculated data obtained from TPS. In general, the measured dose by the MOSFET detector is lower than the calculated doses at the ICRU reference points. This can be explained to a large extent with the steep dose gradient and positioning error of in vivo probes at the ICRU reference point. However, in spite of inaccuracy in the placement of MOSFET, the measured dose values can help prevent large errors from occurring during dose delivery.

Sha et al. [13] measured the rectum dose delivered in intracavitary application with 0.1 $\mathrm{cm}^{3}$ ionization chamber probe and compared with ICRU reference point dose calculated by TPS. Their results for the 86 patients evaluated prospectively showed that the difference between the measured and TPS calculated dose was less than 5\%, 5-10\% and $10-14 \%$ in 52, 26 and 8 patients. Although, in our study, the difference between the MOSFET dose and TPS calculated dose was within $\pm 5 \%, \pm$ $5-10 \%$ and $\pm 10-15 \%$ in 31,8 and 7 patients, respectively. Four patients recorded dose deviation exceeding $\pm 15 \%$.

Sakata et al. [14] evaluated the dose to rectum using a semiconductor detector in 105 patients treated with HDR brachytherapy. Their 
Rectal and Bladder Dose Measurements

results showed that the deviation between planned and measured doses is 5\% for $30.8 \%$ of the cases. These differences reached up to $10 \%$ in $56 \%$ of the cases and up to $20 \%$ in $85 \%$ of the cases. They also observed that the deviation is more than $20 \%$ in $15 \%$ of the patients.

Waldhäusl et al. [15] considered 55 patients of gynecological cancer who were treated with HDR brachytherapy. They measured the dose using diodes. They observed that the difference between the measured dose and calculated dose is from $-31 \%$ to $+90 \%$. These high differences were attributed to the uncertainty in the positioning of detector.

In this study, the described dosimetric procedure allows the evaluation of the dose delivered to the rectum and bladder as a consequence of the HDR brachytherapy for cervix cancer patients. The variation in the MOSFET response for the angles $0^{\circ}, 90^{\circ}, 180^{\circ}$ and $270^{\circ}$ was found to be $\pm 2 \%$. This study closely is consistent with the study carried out by Ramaseshan et al. [16] using micro MOSFET where the angular dependence was found to be $\pm 2 \%$ in a cylindrical phantom. Such type of procedure (online dosimetry) is very useful when the radiation dose is delivered for cervix cancer patients for comparing with the TPS delivered dose. With this approach, we can reduce the large errors between the planned and measured dose in order to protect the organ and minimize complications that may occur when rectum is exposed to high radiation doses.

\section{Conclusion}

In this study, bladder and rectum dose was estimated by MOSFET dosimeter and compared to that from TPS. In summary, the dose calculated by TPS was larger than the dose measured by MOSFET. The differences between measured and calculated doses seem to arise due to positional changes of anatomical organs and MOSFET during simulation and treatment delivery procedures. The use of a small size MOSFET dosimeter is an effective tool for measuring accurate dose in high-dose gradient regions around brachytherapy sources, as in majority of cases the dose deviation was within $\pm 5 \%$. Therefore, to reduce the risk of dose delivery errors in brachytherapy, invivo dosimetry should be performed as a quality assurance procedure.

\section{Acknowledgment}

Authors would like to thank Ms. Shanta R. Lakshmi from Team Best Thretaronics Asia Pvt. Ltd., Chennai, India.

\section{Conflict of Interest}

None

\section{References}

1. Perez CA, Camel HM, Kuske RR, Kao MS, Galakatos $A$, Hederman MA, et al. Radiation therapy alone in the treatment of carcinoma of the uterine cervix: a 20-year experience. Gynecol Oncol. 1986;23:12740. doi: 10.1016/0090-8258(86)90216-7. PubMed PMID: 3943757.

2. Thompson S, Delaney G, Gabriel GS, Jacob $S$, Das P, Barton M. Estimation of the optimal brachytherapy utilization rate in the treatment of carcinoma of the uterine cervix: review of clinical practice guidelines and primary evidence. Cancer. 2006;107:2932-41. doi: 10.1002/cncr.22337. PubMed PMID: 17109449.

3. Chassagne D, Dutreix A, Almond P, Burgers J, Busch M, Joslin C. Dose and volume specification for reporting intracavitary therapy in gynaecology. ICRU Report 38; Bethesda: ICRU; 1985.

4. Haie-Meder C, Pötter R, Van Limbergen E, Briot E, De Brabandere M, Dimopoulos J, et al. Recommendations from Gynaecological (GYN) GEC-ESTRO Working Group (I): concepts and terms in $3 \mathrm{D}$ image based 3D treatment planning in cervix cancer brachytherapy with emphasis on MRI assessment of GTV and CTV. Radiother Oncol. 2005;74:23545. doi: 10.1016/j.radonc.2004.12.015.

5. Pötter R, Haie-Meder C, Van Limbergen E, Barillot I, De Brabandere M, Dimopoulos J, et al. Recommendations from gynaecological (GYN) GEC ESTRO working group (II): concepts and terms in 3D image-based treatment planning in cervix cancer brachytherapy-3D dose volume parameters and aspects of 3D image-based anatomy, radiation physics, radiobiology. Radiother Oncol. 2006;78:67-77. 
doi: 10.1016/j.radonc.2005.11.014.

6. Kim RY, Shen S, Duan J. Image-based threedimensional treatment planning of intracavitary brachytherapy for cancer of the cervix: dose-volume histograms of the bladder, rectum, sigmoid colon, and small bowel. Brachytherapy. 2007;6:187-94. doi: 10.1016/j.brachy.2006.11.005.

7. International Commission on Radiation Units and Measurements. ICRU report 58: Dose and volume specification for reporting interstitial therapy; Bethesda: ICRU; 1997.

8. Stuecklschweiger GF, Arian-Schad K, Poier E, Poschauko J, Hackl A. Bladder and rectal dose of gynecologic high-dose-rate implants: comparison of orthogonal radiographic measurements with in vivo and CT-assisted measurements. Radiology. 1991;181:889-94. doi: 10.1148/radiology.181.3.1947116.

9. Lahtinen T, Tenhunen M, Vayrynen M. ICRU reference points and maximum doses of rectum and bladder in intracavitary radiotherapy. Radiother Oncol. 1993;28:174-6. doi: 10.1016/01678140(93)90011-v. PubMed PMID: 8248560.

10. Clark BG, Souhami L, Roman TN, Evans MD, Pla C. Rectal complications in patients with carcinoma of the cervix treated with concomitant cisplatin and external beam irradiation with high dose rate brachytherapy: a dosimetric analysis. Int J Radiat Oncol Biol Phys. 1994;28:1243-50. doi: 10.1016/0360-3016(94)90501-0. PubMed PMID: 8175412.
11. Alecu R, Alecu M. In-vivo rectal dose measurements with diodes to avoid misadministrations during intracavitary high dose rate brachytherapy for carcinoma of the cervix. Med Phys. 1999;26:768-70. doi: 10.1118/1.598598. PubMed PMID: 10360540.

12. Waldhäus। C, Wambersie A, Pötter R, Georg D. In-vivo dosimetry for gynaecological brachytherapy: physical and clinical considerations. Radiother Oncol. 2005;77:310-7. doi: 10.1016/j.radonc.2005.09.004.

13. Sha RL, Reddy PY, Rao R, Muralidhar KR, Kudchadker RJ. Evaluation of rectal dose during highdose-rate intracavitary brachytherapy for cervical carcinoma. Med Dosim. 2011;36:377-82. doi: 10.1016/j.meddos.2010.09.006. PubMed PMID: 21144733.

14. Sakata $K$, Nagakura $H$, Oouchi A, Someya M, Nakata $K$, Shido $M$, et al. High-dose-rate intracavitary brachytherapy: results of analyses of late rectal complications. Int $J$ Radiat Oncol Biol Phys. 2002;54:1369-76. doi: 10.1016/s03603016(02)03055-9. PubMed PMID: 12459359.

15. Waldhäus। C, Wambersie A, Pötter R, Georg D. In-vivo dosimetry for gynaecological brachytherapy: physical and clinical considerations. Radiother Oncol. 2005;77:310-7. doi: 10.1016/j.radonc.2005.09.004.

16. Ramaseshan $R$, Kohli $K$, Zhang $T$, Lam T, Norlinger $B$, Hallil $A$, et al. Performance characteristics of a microMOSFET as an in vivo dosimeter in radiation therapy. Phys Med Biol. 2004;49:4031-48. 